\title{
Panorama da percepção dos acadêmicos frente a ensino-aprendizagem no contexto da pandemia
}

\author{
Marcela Costa de Almeida Silva ${ }^{1}$ \\ Juan Felipe Galvão da Silva² \\ Geovanna Ribeiro Athie ${ }^{3}$ \\ Enzo Lustosa Campos ${ }^{4}$ \\ Ana Paula da Silva Perez ${ }^{5}$
}

\section{RESUMO:}

Diante da pandemia da COVID-19, a suspensão de atividades presenciais no âmbito da educação foi necessária para promover medidas de prevenção contra essa enfermidade. Com a demanda de realizar isolamento social e a manutenção da educação, o ensino passou por muitas mudanças. Sendo assim, compreender a percepção dos alunos diante desse novo cenário de ensino-aprendizagem se faz necessário para avaliar as principais problemáticas enfrentadas. Diante disso, realizou-se revisão de literatura do tipo umbrella review, na qual foram analisadas matérias em veículos de mídias e 25 trabalhos na base PubMed, 20 no banco de dados Scielo e 62 artigos na BVS, desses, foram selecionados 11,9 e 15, respectivamente. Os resultados demonstram que os empecilhos perpassam o campo das limitações de acesso à internet e tecnologias. Portanto, conhecer a realidade enfrentada pelos alunos mostra-se necessário para mitigar os impactos da pandemia na aprendizagem. Palavras-chave: Pandemia. Ensino. Educação.

\section{Students' perception panorama of the of teaching-learning in the context of the pandemic}

\begin{abstract}
:
In view of the COVID-19 pandemic, the suspension of on-site activities in the field of education was necessary to promote preventive measures against this disease. With the demand for social isolation and the maintenance of education, teaching has undergone many changes. Seeing the above, understanding the perception of students in the face of this new teaching-learning scenario is necessary to assess the main problems faced. Therefore, a literature review of the umbrella review type was performed, in which articles in media vehicles and 25 works in the PubMed database, 20 in the Scielo database and 62 articles in the VHL were analyzed, of which 11,9 and 15 , respectively. The results demonstrate that the obstacles pervade the field of limitations of internet access and technologies. Therefore, knowing the reality faced by students is necessary to mitigate the impacts of the pandemic on learning.
\end{abstract}

Keywords: Pandemic. Teaching. Education.

\footnotetext{
${ }^{1}$ Acadêmica de Medicina na Universidade Federal de Jataí (UFJ). E-mail: marcelacosta@ discente.ufj.edu.br

2 Acadêmico de Medicina na Universidade Federal de Jataí (UFJ). E-mail: juanfelipe@ discente.ufj.edu.br

${ }^{3}$ Acadêmica de Medicina na Universidade Federal de Jataí (UFJ). E-mail: geovannaathie@ discente.ufj.edu.br

${ }^{4}$ Acadêmico de Medicina na Universidade Federal de Jataí (UFJ). E-mail: enzolustosa@ discente.ufj.edu.br

${ }^{5}$ Docente do curso de Medicina na Universidade Federal de Jataí (UFJ), doutora em Biologia Celular e Estrutural.

E-mail: paulabio_perez@ufj.edu.br
} 


\section{INTRODUÇÃO}

O cenário pandêmico, relacionado à doença causada pelo novo coronavírus (SARSCoV-2), exigiu das autoridades sanitárias em diversas nações, medidas para evitar o contágio e a rápida disseminação desse agente (LIMA et al, 2020). Em consequência disso, práticas como o distanciamento social, manutenção do funcionamento restrito a atividades essenciais e, sobretudo, o fechamento de universidades e outros centros de ensino foram adotadas (EL KHATIB, 2020; LIMA et al., 2020). No Brasil, cerca de 182.600 instituições suspenderam as aulas presenciais, deixando 47 milhões de alunos brasileiros no ensino fundamental, médio e ensino superior sem aulas. Como 850 milhões de alunos em 191 países, eles tiveram que se adaptar a uma nova realidade de classes remotas ou sem classes (MUSTAFA, 2020).

Como alternativa a essa situação, ocorreu a implantação, ao nível nacional, de metodologias de ensino à distância, que, mesmo sendo realidade no país em contextos pregressos, tornou-se notória a discussão sobre a efetividade e os desafios desse método (BACAN; MARTINS; SANTOS, 2020). Diante disso, diversas plataformas passaram a ser utilizadas de modo a proporcionar a interação, o aprendizado e a capacitação de discentes, em variadas graduações, como o Google Meet, o Classroom, aplicativos do Google para apresentações, dentre outros (CAMACHO, 2020; LIMA et al., 2020).

O debate, contudo, sobre a igualdade do acesso e os recursos não só materiais, como também psicológicos, relacionados a esse modo de ensino apresentou significativas constatações sobre as deficiências no ensino-aprendizado, além do reforço a inequidades sociais preexistentes (PINTO, 2020). Assim, discute-se a abrangência e os impactos da adoção dessas propostas tecnológicas, mesmo que em caráter emergencial, para substituição de metodologias presenciais na educação, nos variados níveis, do Ensino Básico ao Ensino Superior (MENDONÇA, 2020).

Posto isso, é fundamental compreender como os acadêmicos lidam com esse cenário e, sobretudo, a percepção destes quanto ao ensino-aprendizagem na realidade pandêmica, de modo a evidenciar experiências exitosas e possíveis demandas a serem superadas.

Por meio deste estudo, pretende-se abordar a percepção dos discentes diante dos processos de ensino e aprendizagem durante a pandemia da COVID-19. Nesse esteio, esperase analisar o panorama nacional dos acadêmicos frente aos novos desafios ocasionados pela emergência em saúde pública iniciada em dezembro de 2019, com os primeiros casos dessa enfermidade registrados no Brasil em 2020. Ademais, objetiva-se analisar as adaptações e os recursos destinados à adequação de métodos de ensino tradicionais ao modelo de ensino virtual, 
independente do formato implantado, educação à distância ou ensino remoto. Desse modo, espera-se compreender as diversas variáveis relacionadas a essas mudanças no que diz respeito à sistemática de ensino-aprendizagem, de maneira a englobar aspectos socioeconômicos, demográficos e psicossociais na avaliação dessa temática.

\section{METODOLOGIA}

O presente estudo consiste em uma revisão de literatura do tipo umbrella review (GRANT, 2009), que se refere ao levantamento e análise de evidências sobre um tema amplo e genérico a partir de publicações variadas e acessíveis, realizada com o intuito de avaliar o panorama de percepção dos acadêmicos frente ao ensino-aprendizagem no contexto da pandemia. Diante disso, para a pesquisa, foram analisadas matérias em veículos de comunicação e foram selecionados 44 artigos das principais bases de dados, PubMed, Biblioteca Virtual em Saúde (BVS) e Scielo. Nesse sentido, utilizaram-se os Descritores em Saúde "Brazil", "Distance education" e "COVID-19"; além de empregar o operador booleano “AND" entre os termos mencionados. A partir disso, foram encontrados 25 trabalhos na base PubMed, 20 no banco de dados Scielo e 62 artigos na BVS, dos quais foram selecionados 11, 9 e 15 , respectivamente.

Como critérios de inclusão, adotou-se o ano de publicação, de modo a considerar apenas pesquisas entre 2020 e março de 2021; abordagem da questão relacionada à percepção dos acadêmicos quanto ao ensino na realidade pandêmica; textos adequados à proposta temática pretendida. Ademais, como critérios de exclusão, consideraram-se os seguintes aspectos: eliminação de metanálises; abordagens restritas a determinados cursos de graduação; limitação do estudo a um único centro universitário ou região.

\section{RESULTADOS E DISCUSSÃO}

A adaptação ao ensino superior já é naturalmente um processo multifacetado, envolvendo diversos desafios intrapessoais, interpessoais e ambientais, que exigem o necessário desempenho de novos papéis para ajustamento ao novo contexto, bem como agir de forma proativa em relação à carreira escolhida (ALMEIDA; SOARES, 2004; ASTIN, 1993). Segundo a UNESCO, com a declaração da situação pandêmica provocada pelo coronavírus, ao menos 85 países ao redor do mundo fecharam suas escolas e universidades, e suspenderam suas atividades presenciais (AGÊNCIA BRASIL, 2020). 
Cerca de 33.000 domicílios foram base de estudo sobre o Uso das Tecnologias da Informação e Comunicação nas Famílias Brasileiras pelo estatutário Comitê Gestor da Internet no Brasil (2019). Os resultados revelaram que a ligação à Internet ainda não é universal, apresentando uma piora nas zonas rurais, onde apenas $44 \%$ dessas famílias têm acesso à Internet., Ressaltase ainda, algumas considerações sobre os desafios no ensino remoto, tais como: a disponibilidade efetiva da internet e de outros recursos tecnológicos que possibilitem a ocorrência do ensino remoto; a capacitação (docente e discente) constante diante da velocidade em que os recursos tecnológicos são modificados e disponibilizados; a importância da interação entre os docentes no transcurso da disciplina para que a mesma possa ocorrer de forma visível ao aluno de acordo com o planejamento remoto disposto no início; disponibilidade docente para constante feedback ao discente que coadune com a proposta da aula ministrada, visando um aprendizado colaborativo; a visibilidade do cuidado com o outro, por meio de um aprendizado integrado e cooperativo no âmbito do ensino remoto (CAMACHO, 2020).

Nesse viés, da limitação digital, a falta de autonomia do aluno, ambientes domésticos inadequados para estudo e as dificuldades de concentração são fatores que resultam na descontinuidade no processo de aprendizagem. Para muitos alunos que precisam trabalhar e estudar, alterações nas jornadas de trabalho causadas pelos frequentes decretos municipais e estaduais, a fadiga física e emocional adicional que afeta aqueles que ainda estão trabalhando, além do alto impacto na saúde mental dos estudantes causados ou agravados pelo isolamento social, exigem adaptações tanto das instituições como social para tentar amenizar a situação (SAHU, 2020).

Em suma, a descontinuidade nos horários de aulas, o atraso no calendário das instituições públicas de ensino, a impossibilidade de realização de atividades práticas, e diversas outras incertezas ocasionadas pela pandemia tem um forte impacto sobre os alunos e, principalmente, na qualidade de sua formação. Sendo que esta, é agravada com a falta de previsões e de um planejamento local, regional e de âmbito nacional.

Portanto, o ensino remoto deve ser inclusivo, propondo ações e medidas que visem assegurar melhorias na qualidade do ensino, com investimentos na capacitação docente e na ruptura de barreiras atitudinais. O momento propicia novas experiências e desafios com pleno entendimento de que o ensino remoto nos permite reflexões sobre a complexidade futura diante da pandemia da COVID-19 (CAMACHO, 2020). 
É importante ressaltar, que a pandemia mudou totalmente a realidade dos estudantes, pois, muitas vezes, tiveram que lidar com doença e/ou morte de amigos e familiares, gerando impactos psicoemocionais que podem permanecer pela vida toda. Mesmo em situações de luto, muitos alunos viram suas rotinas mudadas e/ou tiveram que assumir responsabilidades familiares (SAHU, 2020; VIEIRA, 2020).

Por isso, é de extrema importância salientar as limitações e dificuldades enfrentadas pelos professores e alunos, visto que desde o início da pandemia, inúmeras pessoas tiveram suas vidas mudadas. Diante disso, o processo ensino-aprendizagem dos acadêmicos deve ser flexível, cabendo a cada realidade entender e saber e tentar contornar, principalmente pelo Brasil estruturar desigualdades ainda vivenciadas de forma ativa no país.

\section{CONSIDERAÇÕES FINAIS}

O cenário posto pela pandemia da COVID-19 pôs à prova o sistema educacional. Diante dessa situação diversos problemas emergiram, impactando diretamente o ensino-aprendizagem dos alunos. Questões técnicas como o acesso a tecnologias, conectividade à internet $\mathrm{e}$ habilidade tanto dos alunos quanto dos docentes a lidar com as plataformas de ensino interferem diretamente no aprendizado. Ademais, medidas de isolamento social tornaram a casa o reduto do ensino, e que muitas vezes não é um ambiente adequado para tal. É notório os impactos psíquicos e sociais da pandemia, por vezes, precisam-se lidar individualmente, com sentimentos negativos e situações sem precedentes.

Portanto, conhecer a realidade enfrentada pelos alunos, pode ser uma alternativa para auxiliar a comunidade estudantil/acadêmica a lidar com os empecilhos postos pelo contexto pandêmico, tal medida pode ser inspirada nas estratégias levantadas pela Organização Mundial da Saúde (OMS) para mitigar a fadiga pandêmica, em um cenário que é preciso manter medidas de prevenção para a COVID-19 e que a população já está acometida pelo tempo e à proporção que a pandemia vem perdurando. 


\section{REFERÊNCIAS BIBLIOGRÁFICAS}

ANDRADE, Raíssa Bárbara Nunes Moraes; ZERBINI, Thaís. - Estilos e estratégias de aprendizagem em educação a distância: diferenças e semelhanças conceituais - Learning styles and learning strategies in distance education: conceptual differences and similarities - Estilos y estrategias de aprendizaje en la educación a distancia: diferencias y similitudes conceptuales Rev. psicol. organ. trab;20(3): 1150-1156, jul.-set. 2020. tab

BACAN, Aline Ribeiro; Martins, Gustavo Henrique; SANTOS, Acácia Aparecida Angeli dos. - Adaptação ao Ensino Superior, Estratégias de Aprendizagem e Motivação de Alunos EaD Adaptation to Higher Education, Learning Strategies and Motivation of DL Students Adaptación a la Educación Superior, Estrategias de Aprendizaje y Motivación de Alumnos EaD - Psicol. ciênc. prof;40: e211509, jan.-maio 2020.

BENINI, Elcio Gustavo; FERNANDES, Maria Dilnéia; PETEAN, Gustavo Henrique; Penteado, Raphael Camargo; MAGNIN, Luana Silvy de Lorenzi Tezza. - Educação a distância na reprodução do capital: entre a ampliação do acesso e a precarização e alienação do trabalho docente - Distance education in the reproduction of capital: between the expansion of access and the precariousness and alienation of teaching work - Educación a distancia en la reproducción del capital: entre la ampliación del acceso y la precarización y alienación del trabajo docente - Trab. educ. saúde;18(3): e00307139, 2020.

BRASIL. Paulo Meyer Nascimento. Ipea. Acesso domiciliar à internet e ensino remoto durante a pandemia. Brasília. 2020. Disponível em: http://dx.doi.org/10.38116/ntdisoc88. Acesso em: 03 de mar. 2020.

CAETANO, Rosângela; SILVA, Angélica Baptista; GUEDES, Ana Cristina Carneiro Menezes; PAIVA, Carla Cardi Nepomuceno de; RIBEIRO, Gizele da Rocha; SANTOS, Daniela Lacerda; SILVA, Rondineli Mendes da. - Challenges and opportunities for telehealth during the COVID-19 pandemic: ideas on spaces and initiatives in the Brazilian context. Desafios e oportunidades para telessaúde em tempos da pandemia pela COVID-19: uma reflexão sobre os espaços e iniciativas no contexto brasileiro. - Cad Saude Publica;36(5): e00088920, 2020. 
CAMACHO, Alessandra Conceição Leite Funchal. - Ensino remoto em tempos de pandemia da covid-19: novas experiências e desafios - Remote teaching in times of the covid-19 pandemic: new experiences and challenges - Educación remota en tiempos de pandemia del covid-19: nuevas experiencias y desafíos - Online braz. j. nurs. (Online);19(4): [1-4], dez. 2020.

CARVALHO, Vitor Oliveira; CONCEIÇÃO, Lino Sergio Rocha; GOIS JR, Miburge Bolivar. COVID-19 pandemic: Beyond medical education in Brazil. Journal of Cardiac Surgery, v. 35

n. 6

6, p.

1170 ,

2020 .

CHINELATTO, Lucas Albuquerque; COSTA, Thamara Rodrigues da; MEDEIROS, Vitor Macedo Brito; BOOG, Gustavo Henrique Pereira; HOJAIJ, Flávio Carneiro; TEMPSKI, Patricia Zen; MARTINS, Milton de Arruda. What You Gain and What You Lose in COVID19: perception of medical students on their education. Clinics, [S.L.], v. 75, p. 1-3, 2020. Fundacao Faculdade de Medicina. http://dx.doi.org/10.6061/clinics/2020/e2133.

CORNIDE-REYES, Hector et al. A multimodal real-time feedback platform based on spoken interactions for remote active learning support. Sensors, v. 20, n. 21, p. 6337, 2020.

EL KHATIB, A. S.; CHIZZOTTI, A. Videoconferencing classes: A solution to the social distance caused by COVID-19 or a big problem? Revista Edapeci-Educacao a Distancia E Praticas Educativas Comunicacionais E Interculturais, p. 26-45, 2020.

GONÇALVES, Patrícia Palmeira; SILVA, Claudia Neves da. - Educação a distância e formação profissional do/da assistente social: elementos para o debate - Revista Katálysis; 23(1); 90-100; 2020-04

GRANJEIRO, Érica Maria et al. Estratégias de ensino à distância para a educação interprofissional em Saúde frente à pandemia COVID-19. Revista de Divulgação Científica Sena Aires, v. 9, p. 591-602, 2020. 
GRANT, Maria J.; BOOTH, Andrew. A typology of reviews: an analysis of 14 review types and associated methodologies. Health Information \& Libraries Journal, [S.L.], v. 26, n. 2, p. 91-108, 27 maio 2009. Wiley. http://dx.doi.org/10.1111/j.1471-1842.2009.00848.x

LELLIS-SANTOS, Camilo; ABDULKADER, Fernando. Smartphone-assisted experimentation as a didactic strategy to maintain practical lessons in remote education: alternatives for physiology education during the COVID-19 pandemic. Advances in physiology education, v. 44, n. 4, p. 579-586, 2020.

LIMA, Alisson Cunha et al. Desafios da aprendizagem remota por estudantes universitários no contexto da Covid-19. Revista de Divulgação Científica Sena Aires, v. 9, p. 610-617, 2020.

LIMA, Karine Ramires et al. Student assessment of online tools to foster engagement during the COVID-19 quarantine. Advances in physiology education, v. 44, n. 4, p. 679-683, 2020.

MACHADO, Maria Beatriz de Sá Dias. On the Frontlines, Behind the Computer Screen. Academic Medicine, [S.L.], v. 95, n. 11, p. 10-11, 27 out. 2020. Ovid Technologies (Wolters Kluwer Health). http://dx.doi.org/10.1097/acm.0000000000003601.

MAGALHÃES, Amanda Júlia de Arruda et al. O ensino da anamnese assistido por tecnologias digitais durante a Pandemia da Covid-19 no Brasil. Revista Brasileira de Educação Médica, v. 44, 2020.

MARTIN, Alice et al. Continuing medical and student education in dermatology during the coronavirus pandemic-a major challenge. JDDG: Journal der Deutschen Dermatologischen Gesellschaft, v. 18, n. 8, p. 835-840, 2020.

MATTAR, João; RODRIGUES, Lucilene Marques Martins; CZESZAK, Wanderlucy; GRACIANI, Juliana. - Competências E Funções Dos Tutores Online Em Educação A Distância - Educação Em Revista; 36();2020. 
MENDONÇA, José Ricardo Costa de et al. Políticas públicas para o Ensino Superior a Distância: um exame do papel da Universidade Aberta do Brasil. Ensaio: avaliação e políticas públicas em Educação, v. 28, p. 156-177, 2019.

OLIVEIRA ARAÚJO, Francisco Jonathan et al. Impact of Sars-Cov-2 and its reverberation in global higher education and mental health. Psychiatry research, v. 288, p. 112977, 2020.

PELOSO, Renan Morais et al. Notes from the field: concerns of health-related higher education students in Brazil pertaining to distance learning during the Coronavirus pandemic. Evaluation \& the Health Professions, v. 43, n. 3, p. 201-203, 2020.

PINTO, Francisco Ricardo Miranda et al. COVID-19: A new crisis that reinforce inequality in higher education in Brazil. Disponível em: https://preprints.scielo.org/index.php/scielo/preprint/view/1341.

PRATA, Juliana Amaral; MELLO, Alex Simões de; SILVA, Frances Valéria Costa e; FARIA, Magda Guimarães de Araújo. Pedagogical mediations for non-formal nursing teaching during the COVID-19 pandemic. Revista Brasileira de Enfermagem, [S.L.], v. 73, n. 2, p. 1-5, 2020. FapUNIFESP (SciELO). http://dx.doi.org/10.1590/0034-7167-2020-0499.

RODRIGUES, Bráulio Brandão et al. Aprendendo com o Imprevisível: Saúde mental dos universitários e Educação Médica na pandemia de Covid-19. Revista Brasileira de Educação Médica, v. 44, 2020.

SANTOS, Bruna Mascarenhas; CORDEIRO, Maria Eduarda Coelho; SCHNEIDER, Ione Jayce Ceola; CECCON, Roger Flores. - Educação Médica durante a Pandemia da Covid-19: uma Revisão de Escopo - Medical Education during the Covid-19 Pandemic: a Scoping Review - Rev. bras. educ. méd;44(supl.1): e139, 2020. tab, graf

SILVA, Andrey Ferreira da; ESTRELA, Fernanda Matheus; LIMA, Nayara Silva; ABREU, Carlos Tibúrcio de Araújo. - Saúde mental de docentes universitários em tempos de pandemia - Mental health of university professors in times of pandemic - Physis (Rio J.);30(2): e300216, 2020. 
SILVA, Paulo Goberlânio de Barros et al. Distance learning during social seclusion by COVID19: improving the quality of life of undergraduate dentistry students. European Journal of $\begin{array}{lllllll}\text { Dental Education, } & \text { v. } & 25, & \text { n. } & 1, & \text { p. } & 124-134,\end{array}$ SILVA, Tatiana de Paula Santana da et al. Tele-education applied to human communication health to cope with triple epidemics in the state of Pernambuco, Brazil: an experience report. Revista Cefac, v. 22, 2020.

TORRES, Ana Catarina Moura; ALVES, Lynn Rosalina Gama; COSTA, Ana Caline Nóbrega da. Education and health: reflections on the university context in times of COVID-19. In: Education and Health: reflections on the university context in times of COVID-19. 2020.

UMEKAWA, Elienay Eiko Rodrigues; ZERBINI, Thaís. Estratégias de aprendizagem na educação a distância: revalidação de uma escala. Psico, v. 51, n. 2, p. e29638-e29638, 2020.

WANDER, Brenda; GOMES, Marta Quintanilha; PINTO, Maria Eugênia Bresolin. Avaliação da interação em fóruns de discussão na especialização de preceptoria em Medicina de Família e Comunidade a distância. Interface-Comunicação, Saúde, Educação, v. 24, 2020. 